Pub. Mat. UAB

№ 19 Maig 1980

Actas II Congreso de Ecuaciones Diferenciales y Aplicaciones- Valldoreix, Mayo 1979.

RF SULIADOS Y METODOS SOBRE LA PROPIEDAD DE EXTINCION EN TIEMPO FINITO PARA ECUACIONES DE EVOLUCION.

\author{
Por J. IIdefonso Díaz Díaz \\ Dpto. Se Holutiones Funcionales \\ Universid a complutenste de Madrid.
}

91. Introducción.

Daclo el problema de evolución

$$
P \equiv\left\{\begin{array}{l}
\text { Ecuación diferencial de evolución (sobre } x \text { espa } \\
\text { cio de Banach y } \in(0, \infty)) \\
+ \text { Condiciones iniciales, }
\end{array}\right.
$$

diremos que se satisface la propiedaơ de extinciön en tiempo finito si la solución $u$ de $p$ es tal que existe $T_{0}<+\infty$ tal que $u(t)=0$ (en $X)$ para todo $t \geq T_{0}$.

Es natural que el lector desconfie de la aparición de esta propiedad para una clase "amplia" de problemas, pues existen re sultados tajantes (y bien conocidos) que impiden que se produzca la extinciôn en tiempo finito. Recordemos, esquemáticamente, algunos de estos criterios negativos:

* Conservación de alguna "energía". (p.e. $\|u(t)\|_{L}=$ $\left.=\|u(u)\|_{L^{p}}\right)$. 
- Caso de las ecuaciones de Schrodinger y de Klein-Gordon.

- Caso de las ecuaciones hiperbólicas lineales sí mêtricas. (Lis obtiene a partir del tưrema de Stone de generación de grupos unitarios).

* Principio fuerte del mínimo de Nirenberg.

- Caso de ecuaciones lineales uniformemente parabólicas. (Aplíquese a datos $\geq 0$ ).

* Propiedad de "Eorward and backward unique contul.. 1 ion".

- Caso de ecuaciones lineales gobernadas por un semi-grtpo analítico. VVéanse los trabajos de Yosida, Mizohata, etc...)

Sin emisargo, hurgando entre los problemas Iineales, es posible encontrar algunos casos concretos donde aparece la extinciôn en t.f.:

Ejemplo 1. (Díaz [12]). Dado $\Omega$ abierto regular acotado de $\mathbb{R}^{N}$, consideremos el problema

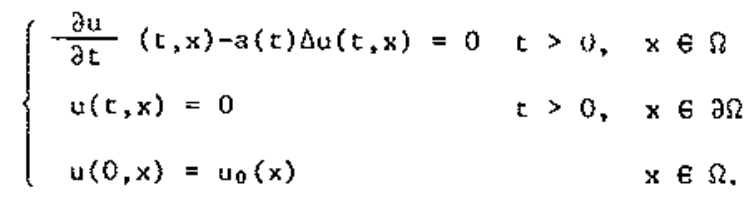

Entonces, si se supone

$$
\begin{gathered}
\exists \mathrm{T}_{0}>0 \text { tal que } \int_{0}^{\mathrm{T}_{\infty}} \mathrm{a}(\mathrm{s}) \mathrm{ds}=+\infty \\
\Delta_{0}+u_{0}=0 \text { en } \Omega \text { y } u_{0}=0 \text { en } \partial \Omega,
\end{gathered}
$$




\section{1a furıción}

$$
u(t, x)= \begin{cases}\exp \left(-\int_{0}^{t} a(s) d s\right) \cdot u_{0}(x) & \underline{s i} 0 \leq t<T_{0} \\ 0 & \underline{s i} \quad t \geq T_{0}\end{cases}
$$

es sulución del ancerior problema. \#

Ejemplo 2, (Majda [2l]). Dado $\Omega$ abierro regular de $\mathbb{R}^{N}$, el proble ma

$$
\begin{cases}u_{t t}-\Delta u+c(x) \cdot u_{t}=0 \quad t>0, & x \in \Omega \\ \frac{\partial u}{\partial n}+Y(x)_{u_{t}}+o(x)_{u}=0 t>0, & x \in \partial \Omega \\ u(0, x)=u_{0}(x) & x \in \Omega \\ u_{t}(0, x)=u_{1}(x) & x \in \Omega\end{cases}
$$

(supuestos c, $\sigma$ y $Y$ funciones $\geq 0$ y $<$ con soporte compacto) posee extinción en $t \cdot f \leftrightarrow \leftrightarrow r(x) \geq 1 \stackrel{\text { c.p.t }}{t} x \in \partial \Omega$ J

l.a situación es diferente cuando el problema $P$ es no

lineal. Como expondremos a continuación, existe una "amplia" clase de estos problemas para los que tiene la citada propiedad. Algunos de elloṣ tienen una importante relevancia física (véase mâs tarde el problema (4) que aparece con la "difusión de una partícula a través de un campo magnético en un plasma toroidal multipolo".

En lo que sigue de exposición, se harâ más hincapié en los métodos existentes para obtener la propiedad que en los resultados. (Un enfoque diferente puede encontrarse en Diaz [12]). En con creto, el plan serä el siguiente:

§2. Mêtodos de comparaciôn: 
2.1. Vía desigualdades de sobolev.

2.2 . Vía construcción de super y subsulitiones "ad hoc".

§3. Métodos abstraccus.

54. Observaciones finales.

En esencia, §2 se refiere a diversas ecunciones :arabólicas no lineales de 2 o orden y $\$ 3$ a i ucuaciones variacionales pa rabólicas formuladas en términos de operitores acretivos. A fin de una exposición más fuida apenas se laráa alusión a la xistencia y unicidad de la sulución, 10 que se supondrá (y de hecho se tiene) so bre espacios funciolules adecuados. El lector "ortodoxo" puede encon trar esos detalles en bía [12] y/o la bibliografia de dicho trabajo.

§2. Métodos de comparración.

Cono ya se ha dicho, tales métodos utilizan de forma esencia? el hecho de que las ecuaciones en derivadas parciales de $p$

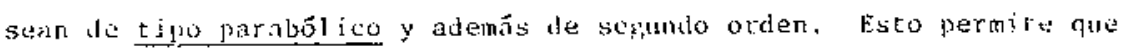
se tenkan propiedales del estilu"principio del máximo y compración de soluciones", al igual que el caso de Ja ecuación lineal del calor. La diterente utilización de escas propintacles conduce a la subclasificaciôn siģuitute:

2.1. Y.al desigualdades die subolev.

consideremos, por ejenpla el probliena

$$
\left\{\begin{array}{lll}
u_{t}-\Delta u^{m}=0 & \text { en } & (1, \cdots) \times \mathbb{R}^{N} \\
u(0, x)=u_{0}(x) \text { en } & & \mathbb{R}^{\text {in }},(11.0)
\end{array}\right.
$$


Siguiendo a Benilan y Crandall [4], multipliqumos por $\frac{1}{\alpha+1} u^{\alpha}$ en la ecuación e integrenos sobre $\mathbb{R}^{N}$. Tras utilizar la fórmula de Green se tendria (Eormalmente) que

$$
\frac{d}{d t} \int_{\mathbb{R}^{N}} u^{u+1}+-\frac{1}{c+1} \int_{\mathbb{R}^{N}} \nabla^{\mathrm{m}} \nabla_{u^{\alpha}}^{\alpha}=0
$$

Tras cálculos, es fácil ver que

$$
\int_{B R} v^{N} v^{m} v^{\alpha}=\frac{4 m i x}{(n+\alpha)^{2}} \int_{\mathbb{R}}\left|\nabla u \frac{m+\alpha}{2}\right| .
$$

Utilizandt) ahora la desigualdad de Sobolev $\left(H^{2}\left(\mathbb{R}^{N}\right) \subset L^{2}{ }^{k}\left(\mathbb{R}^{N}\right)\right.$, $\left.2 *=\frac{2 N}{N-2}\right) e \cdot d$.

$$
\left.\int_{\mathbb{R}} N|\nabla \phi|^{2}\right)^{1 / 2} \geq\left(\because\left(\int_{\mathbb{R}^{N}}\left|\phi^{2 *}\right|\right)^{1 / 2 *}\right.
$$

se obtiene entonces

$$
\int_{\mathbb{R}^{N}} \nabla u^{m} \nabla u^{\alpha} \geq c\left(\int_{\mathbb{R}^{N}} u^{\left.(m+\alpha) \frac{2 \star}{2}\right)^{2 / 2 *}}\right.
$$

(por comodidad las constantes se representan uniformemente por $C$ ). Tomando $\alpha$ de forma que $(m+\alpha) 2 \star / 2=:,+1$ (e.d. $\alpha=\frac{(2-m 2)}{2 \star-2}$ ) y exigiendo que sea positiva (e.d. $0<m<\frac{N-2}{N}$ ), se obtiene finalmente que

$$
\frac{d}{d t}\left(\int_{\mathbb{R}^{N}} u^{\alpha+1}\right)+c\left(\int_{\mathbb{R}^{N}} u^{\alpha+1}\right)^{z / 2 \star} \leq 0
$$

Ahora bien, toda función $f$ verificando la desigualdad

$$
\mathrm{f}^{\prime}+\mathrm{CF}^{Y} \leq 0 \quad(\mathrm{f}:[0, \infty)+[0, \infty), c>0,0 \leq \gamma<1)
$$

tiene la propiedad de que $\exists \mathrm{T}_{0}>0$ tal que $f(t)=0$ para $t \geq \mathrm{T}_{0}$, pues basta comparar $f$ con la función 


$$
g(t)= \begin{cases}a\left(T_{0}-t\right)^{\frac{1}{1-\gamma}} & \text { si } t \leq T_{0}, \quad a=((l-\gamma) c)^{\frac{1}{1-\gamma}} \\ 0 & \text { si } \quad t \geq T_{0} .\end{cases}
$$

En efecto, es claro qute $g$ sitisfact

$$
\mathrm{g}^{\prime}+\mathrm{Cg}=\mathrm{O}
$$

$y$ como $g(0)=$ a $\mathrm{T}_{0}^{\frac{1}{1-\bar{Y}}}$, basta tomax $\mathrm{T}_{0}$ suficientemente grande para que $g(0) \geq E(0) \quad y$ entunces se obtendría que $0 \leq f(t) \leq g(t)$.

Los argumentos anteriores pueden justificarse rigurosạ mente obreniendose:

Teorema 1. (Benilan-Crandall [4]).

$$
\text { Sean } N \geq 3, \quad 0<m<\frac{N-2}{N}, \quad \alpha=\frac{2-m 2^{\star}}{2^{\star}-2} y
$$

uo $\in L^{a+1}\left(\mathbb{R}^{N}\right) \quad O L^{\prime}\left(\mathbb{R}^{N}\right)$. Entonce: : H poblema

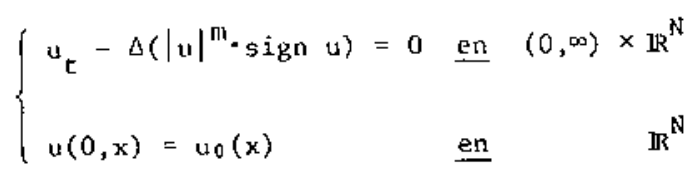

posee una única soluciōn $u \in C\left([0, \infty): \mathrm{L}^{\prime}(\Omega)\right)$, extinguirndose en tiem po finito.

En el anterior trabajo se muestra que si $\frac{N-2}{N}<m \leq 1$ se tiente conservación de la norma $\mathrm{L}^{1}$, por 10 que no puede haber extinción en t.f. Sin embargo, se verá en 2.2 que si se escudia la ecuación de (1) sobre un abierto $\Omega$ acotado de $\mathbb{R}^{N}$ y con condicio- 
nes de Dirichlet, la extinción aparece cuando $0<m<1, \quad\left({ }^{1}\right)$

EI mëtodo auterior ha sidu tumbien iplicado a otras ecuaciones no lineales. Asi. por ejumplo Hanberger [1] ha mostrado Ja propicalad en cuestioñ para lat ceuastion

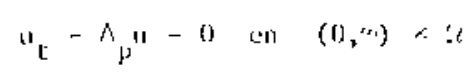

donde

$$
\Delta_{P} u=\sum_{i=1}^{N} \frac{\partial}{\partial x_{i}}\left(\left|\frac{\partial u}{\partial x_{i}}\right|^{p-2} \frac{\partial u}{\partial x_{i}}\right),
$$

$\Omega$ es un abierto acotado $y \frac{2 \mathrm{~N}}{\mathrm{~N}+2}<\mathrm{p}<2$. El caso $\Omega=\mathbb{R}^{\mathrm{N}}$ ha sido tratado por Herrero-Vázquez [19] mostrando que $\forall p>1$ se conserva una cierta norma.

\subsection{Vía construcción de super y subsoluciones "ad hoc".}

En esencia, se trata de construir super y subsoluciones $\bar{u} y \underline{u}$ extinguiéndose en t.f. Asi, los "teoremas de cuuparación" aseguran que

$$
\underline{u}(t, \cdot) \leq u(t, \cdot) \leq \bar{u}(t ; \cdot) \text { para rodo } t \in[0, \infty)
$$

(supuesta u solución del problema en consideraciôn) y de aquí la con clusión. Tales resultados de comparación son característicos de las ecuaciones de 20 orden, y en los problemas no lineales la dificujtad mayor para su obtención reside en la poca regularidad de la solución (Para un resultado tipo vêase p.e., G. Díaz-I. Díaz [9]).

(') Esto también puede obtenerse mediante una utilización de la desi gualdad de Sobolev diferente a la aqui indicada. (Bertiman-Holland [5]). 
A modo de jlustraciōn, construyamos $\bar{u}$ para el proble

ma

$$
\left\{\begin{array}{llr}
u_{t}-\Delta\left(|u|^{n 1} \cdot \operatorname{sign} u\right)=0 & \text { en } & (0, \infty) \times \Omega \\
u=0 & \text { en } & (0, \infty) \times \partial \Omega \\
u(0, x)=u_{0}(x) & \text { en } & \Omega
\end{array}\right.
$$

cuando se supone: $\Omega$ acotado, $0<m<1$ y $u_{0} \in \mathrm{L}^{\infty}(\Omega)$. Sin pérdida de generalidad podemos suponer adenás que $u_{0} \geq 0$. Sea $g(x)$ tal que

y

$$
\exists \lambda>0 \text { cal que } \mathrm{g}+\lambda \circ \mathrm{g}=0 \text { en } \Omega
$$

$$
\exists \theta>0 \text { tal que } 0<\theta \leq \mathrm{g}(\mathrm{x}) \leq 1 \text { en } \bar{\Omega} \text {. }
$$

(tal función puede obtenerse entre los valores propios de $\Delta$ para el problema de Dirichlet sobre $\tilde{\Omega}, \tilde{\Omega} \supset \supset i$ ). Consideremos $f_{0}>0$ suficientemente grande para que

$$
\mathrm{f}_{0} \cdot \theta^{1 / \mathrm{m}} \geq\left\|\mathrm{u}_{0}\right\|_{\mathrm{L}^{\infty}}
$$

sea entonces $f(t)$ la solución de la ec. ordinaria $f^{\prime}(t)+\frac{1}{\lambda} f(t)^{m}=0$ con $f(0)=\hat{E}_{0}$ (gracias a que $0<m<1$ se sabe que $f$ se extingue a partir d. un $\left.\mathrm{T}_{0}<\infty\right)$. Consideremos finalmente $\bar{u}(t, x)=f(t) \cdot(g(x))^{1 / \pi} \cdot$ Se tiene que

$$
\begin{aligned}
& \text { a) } \vec{u}(t, x)=0 \text { para } t \geq T_{0} \\
& \text { b) } \bar{u}(0, x) \geq f_{0} \cdot \theta^{1 / m} \geq\left\|u_{0}\right\|_{L}^{\infty} \geq n_{0}(x) \text { en } \Omega \\
& \text { c) } \bar{u}(t, x) \geq 0 \text { en }(0, \infty) \times \vec{\Omega} \\
& \text { d) } \bar{u}_{t}-A \bar{u}^{m}=E^{\prime}(t) g(x)^{1 / m}-f(t)^{m} \Delta g(x) \geq f^{\prime}(t) g(x)+ \\
& +E(t)^{m}-\frac{g(x)}{\lambda}=g(x)\left(F^{\prime}(t)+\frac{f(t)^{m}}{\lambda}\right)=0 \\
& \text { en }(0, \infty) \times \Omega
\end{aligned}
$$

por lo que $\overrightarrow{\mathrm{u}}$ es efectivamente una supersolución adecuada a nuestro fin. 
Los razonamientos anteriores pueden ser fäcilmente ex tendidos a la ecuas:ión mâs gerieral

$$
u_{\mathrm{L}}-\operatorname{As}(\mathrm{u})=0 \text { en }(0, \cdots) \times: t
$$

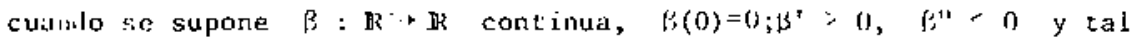
que

$$
\int_{-1}^{1} \frac{d s}{B(s)}<+\infty
$$

supuesto $u_{0} \in L^{\infty}(\Omega)$ (Evans [16]). El siguiente resultado prescinde de coda hipótesis de lerivabilidad sobre $\beta$, es válido para datos uo no necesariamente acotados y además establece la necesidad (ie (4):

Teorenai 2. (G. Díaz - I. Díaz. [9]).

Sea $\Omega$ acotado y uo (p.e.) tal que $u_{0} \in \mathrm{L}^{\mathrm{i}}(\dot{\mathrm{i}})$, con $p>\max \left\{\frac{2 N}{N+2}, N / 2\right\}$. Sea $B: \mathbb{R} \rightarrow \mathbb{R}$ (p.e.), continua no drercecien te tal que $B(0)=0$ y sea u $\in C\left([0, \infty): L^{\prime}(\Omega)\right)$ la solución de

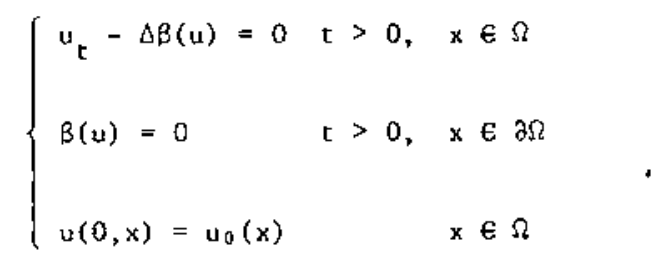

Entonces la condición necesaria y suficiente para que $u$ se extinga en t.f.. es que B satisfaga (4).

La demostración del Teorema 2 se basa en el establecimiento inicial de la misma conclusión para las solusiones del probleกล 


$$
\left\{\begin{array}{lr}
v_{t}+\beta(-\Delta v)=0 t>0, & x \in \Omega \\
v=0 & t>0, \\
v(0, x)=v_{0}(x) & , x \in \partial \Omega
\end{array}\right.
$$

cuando $v_{0} \in L^{\infty}(\Omega)$ ก $H_{0}^{1}(\Omega)$. Por otro lado se muestra que si $\mathrm{u}_{0}=$ $=-\Delta v_{0}$ entonces $u(t, x)=-\Delta v(t, x)$ y ésto junto i ciertos resultados sobre la regularidad de u concluye la prueba. Es de señalar que el Teorema 2 es establecido incluso para $B$ grafo maxímal monótono de $\mathbb{R}^{2}$. verificando $0 \in B(0)$ y $D(\beta)=R(B)=\mathbb{R}\left({ }^{2}\right)$.

Relativo al problema (2) es de destacar el trabajo de Berryman-Holland [S] en el que se estudia minuciosamente el comportamiento de la solución para $t \rightarrow T_{0}$ cuando $0<\mathrm{m}<1$.

Finalizaremos esta sección con dos resultados abstractos aplicables bajo la hipótesis de comparación de soluciones $\Leftrightarrow$ T-acretividad):

\section{Teorema 3 .}

a) (Veron [22]). Sean A ty operador T-acretivo en $L^{1}(\Omega), B$ grafo maximal monótono de $\mathbb{R}^{2}$ con $\beta(0) \exists 0$ y uo $\in L^{\infty}(\Omega)$. Entonces toda solución del problema

$$
\left\{\begin{array}{l}
\frac{d u}{d t}(t)+A(u(t))+B(u(t)) \ni 0 \quad t>0 \\
u(0)=u_{0}
\end{array}\right.
$$

se extingue en t. F. si B satisface (4).

$\left({ }^{2}\right)$ Un resultado para (6) relativo a $\beta$ dependiendo de $x$ y para operadores elipticos de 20 orden măs generales que $-\Delta$ se debe a $G$. Diaz $[B]$. 


\section{b) (I. D1az [13]). Sea A un operador T-acretivo}

en $L^{3}(\Omega) \quad y$ q-lnomogëneo (e.d. $A(\lambda u)=|\lambda|^{q^{-1}} \lambda A(u) \quad \forall \lambda \in \mathbb{R} \quad y$ $\forall u \in v(A)$. Entomes supuesto que $\rho \equiv \mathrm{m} \cdot \mathrm{q}-1$ es $\rho>0$, toda soluciơn del problema

$$
\left\{\begin{array}{l}
\frac{d u}{d t}+A\left(|u|^{n} \cdot \operatorname{sign} u\right)=0 \\
u(0)=u_{0}
\end{array}\right.
$$

satisface

$$
\left.u(t) \geq u(s)\left(\frac{s}{t}\right)^{1 / \rho} \quad \forall t \geq s>0 \quad \text { (supuesto } u_{\theta} \in E^{1}(\Omega), u_{0} \geq 0\right)
$$

$y$ por tanto no puede haber extinción en t.E.

otros criterios positivos para diferentes problemas

perturbados, y negativos para otros operadores homogéneos, pur in encontrarse en los trabajos antes reseñados.

53. Mętodos abstractos.

El problema $P$ de $\$ 1$ puede ser formulado con más prẹ cisión cono

$$
r \equiv\left\{\begin{array}{l}
\frac{d u}{d t}(t)+A(u(t)) \exists f(t), t \in(0, \infty), u(t) \in X, \\
u(0)=u_{0}
\end{array}\right.
$$

siend, $x$ espario de Bumach, $A: D(A)(c x) \rightarrow \mathcal{J}(x)$, f $\in$ ioc $(0, \cdots x)$ $y$ uo $€ X$. En estas tircunstancias es posible dar ilgunos criterios sobre A y sobre $f$ para que la solución u se extinga en t.f.

Este es el caso de operadores A que son multivucus en el origen, situación a la que se llega en el estudio de las Inecuacio- 
nes Variacionales de livolation (B) lectur presle encontrar en Duvat-lions [1S] una formitiz:ión concreca de cstos problemsasi como, algu nos fenómenus físicus en los que aparecersy).

Antes que naxlit, observenters que una comblotón de compati biliats con lia aparicion de la citakta propiedad es que se renga

$\Lambda(0) \ni t(t)$, en $x$ y c.p.t. $t$ suricientemente grande

E) siguitute resultado mustra que la anterior cumbciön es "casi suficiente" cuando ste suponz A multivo'، en el urigen.

Teorema 4. (1. Dïaz (11]).

Sea $A$ m operador abretivo (') en $x$ y $E$ y ${ }^{\prime}$ tales que existe ura unica solución de $P$. Supongamos que existen to y $\quad \rho:\left[t_{0}, \infty\right) \rightarrow \mathbb{R}^{+}$integrable, tales que

$$
\begin{aligned}
& B(f(t)), \rho(t)) \subset A(0) \text { c.p.t. } t \geq t_{B} \\
& \int_{t_{0}}^{\infty} \rho(s) d s=+\infty
\end{aligned}
$$

Entonces Ia sulución (integral) de $P$ se extingu: :n tiempo finito.

\section{Demostración.}

Sin pérdida de generalidad podemos suponer que $u(t) \neq 0$ para todo $t>0$, pues si $\exists t_{1}$ tal que $u\left(t_{1}\right)=0$ entonces la funciôn

( $\left.{ }^{3}\right)$ Definido el producto $\tau(x, y)=1$ im $\frac{\|x+\lambda y\|-\|x\|}{\lambda} \forall(x, y) \in x \times x$,

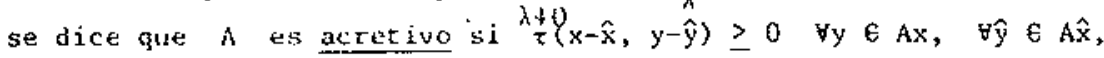
$\forall x, \hat{x} \in D(A)$. 


$$
\tilde{u}(t)=\left\{\begin{array}{lll}
u(t) & \text { si } t<t_{1} \\
0 & \text { si } t \geq t_{1}
\end{array}\right.
$$

sería otra solución de $p$ lo que contradice la unicidad. Gracias a (7) se tiene que $f(s)+\rho(s) \cdot h(s) \in A(0)$ para todo $h(s)$ con $\|h(s)\|_{X} \leq 1 \quad$ c.p.t. $\quad s \in\left(t_{0}, \infty\right)$. Es claro entonces que la Eunción $\hat{u}(t) \equiv 0$ es una solución de $\mathrm{P}$ correspondiente a los datos $\hat{f}(t)=$ $=f(t)+\rho(t) \cdot h(t)$ y $\hat{u}_{0}=0$. Recordando ahota que por la acretivi dad de A se sabe que (Benilan [2])

$$
\|u(t)-\hat{u}(t)\| \leq\left\|u\left(t_{0}\right)-\hat{u}\left(t_{0}\right)\right\|+\int_{t_{0}}^{t} T(u(s)-\hat{u}(s), f(s)-\hat{f}(s)) d s
$$

obtenemos finalmente que

$$
\|u(t)\| \leq\left\|u\left(t_{0}\right)\right\|+\int_{\tau_{0}}^{t} \tau(u(s),-\rho(s) h(s)) d s
$$

Pero es fäcil comprobar que $\tau(x, \lambda y)=\lambda \tau(x, y)$ por lo que se tiene

$$
\|u(t)\| \leq\left\|u\left(t_{0}\right)\right\|-\int_{t_{0}}^{t} \rho(s) \cdot \tau(u(s), h(s)) d s .
$$

Tomando ahora $h(s)=\frac{u(s)}{\|u(s)\|}$ y obserwando que $\tau(x, x)=\|x\|$ se con cluye que

$$
\|u(t)\| \leq\left\|u\left(t_{0}\right)\right\|-\int_{t_{0}}^{t} \rho(s) d s .
$$

Haciendo $t$ suficientemente grande y gracias a $(8)$ se obtiene $u(t)=0$ lo que es una contradicción. /

$$
\text { Las condiciones (7) y (8) obligan a que } A(0) \text { sea topo }
$$
lógicamente grande en $X$. Si se supone $X$ e. de Hilbert el resultado (primeramente establecido en Brezis [6]) es de difícil aplicación salvo para el caso de $X=\mathbb{R}^{\mathrm{N}}$ y entonces $P$ es una ecuación ordina- 
ria multivoca. Sin embargo si se toma. $X=L^{\infty}(\Omega) \quad\left(\Omega\right.$ abierto de $\left.\mathbb{R}^{N}\right)$ son posibles múltiples aplicaciones a problemas en ecuaciones en derivadas parciales. A modo de ilustración expongamos dos ejemplos:

Corolario 1. (I. Diaz [11]).

Sea $B$ un grafo maximal gonótono de $\mathbb{R}^{2}$ tal que $B(0)=\left[\beta^{-}, \beta^{+}\right]$con $\beta^{-}<\beta^{+}$. Sean p.e, uo $\in W^{2},(\Omega)$ y $f \in L_{\text {loc }}^{l}\left(0, \infty: L^{\infty}(\Omega)\right)$ verificando:

(9) $J \varepsilon>0$ y $\exists t_{0} \geq 0$ tales que $B^{-}+\varepsilon \leq f(t, x) \leq B^{+}-\varepsilon$ c.p.t. . $(t, x) \in\left[t_{0}, \infty\right) \times \Omega$. Entonces los siguiente problemas poseen 1.

propiedad de extinciön en t.f.:

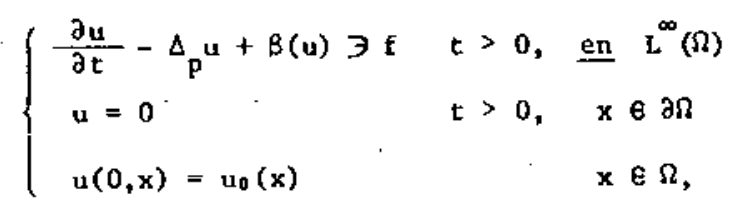

y

$$
\begin{cases}\frac{\partial u}{\partial t}+\beta(-\Delta u) \ni t & t>0, \text { en } L^{\infty}(\Omega) \\ u=0 & t>0, \quad x \in a \Omega \\ u(0, x)=u_{0}(x) & x \in \Omega .\end{cases}
$$

E1 problema (10) había sido antes considerado por varios autores (Brezis-Friedman, Bensoussan-Lions, Evans-Knerr, etc...) para $p=2$ y un caso concreto de $\beta$. Ellos obtenían la extínción en t.f. tomando en (9) $t_{0}=0$ y mostrando a la vez la propiedad de propagación finita de las señales. Esta conjunción de las dos propiedades aparece más en general para (10) cuando se toma $p \geq 2$ y (9) con $t_{0}=0$ (vêase Diaz-Herrero [14]). 
Mediante la relación establecida entre (6) (a(11)) y

en e1 curso de la demostración del teorena 2, es posible formular un resultade de extinción para

$$
\frac{\partial u}{\partial t}-\Delta \beta(u) \exists \mathrm{f} \quad \text { en } \quad(0, \infty) \times \Omega
$$

observemos que ahora la condición de compatibilidad a exigir es que se tenga $-\Delta B(0) \ni f(t, \cdot)$ para $t$ suficientemente grande, o mäs correc tamente

$$
B(0) \geqslant(-A)^{-1}[(t, \cdot) \text { para } t \text { suficientemente grande. }
$$

Cuando $f(0)=0,(12)$ se reduce a exigir que $f(t, \cdot)=0$ para

$t \geq t_{0} y$ en dicho caso el Teorema 2 puede ser fácilmente modificado.

Curituh, B es multívoco en et origen, el corolario l y la rclaciôn men cionada afirman que (12) es "casi suficiente", Leniêndose la extinción en t.f. si

$$
\begin{aligned}
\exists \varepsilon>0 \text { y }] t_{0} \geq 0 \text { tales que } B^{-}+\varepsilon \leq(-A)^{-1} f(t, \cdot) \leq \\
\leq B^{+}-\varepsilon \text { c.p.t. } t \in\left[t_{0}, \infty\right)
\end{aligned}
$$

Como romentario final à esta sección, sẹnalemos que aun que el Teorema 4 no exige la T-acretividad de A sin enbargo hast.: el momento no son conocidas más aplicaciones que las correspondientes a $\mathrm{X}=\mathrm{L}^{\infty}(\Omega)$ y entonces la condición de acretividad estä lijgada de mancra implicite a propiedades de tipo principio del máxino (que caracteri zan a los operadores de $2^{\circ}$ orden). Sería interesarte conocer algún re sultado (por partjcular que éste sea) relittivo a la extinciốn en t.f. para operadores de orden superior a dos. 
54. Observaciones ininil Ls:

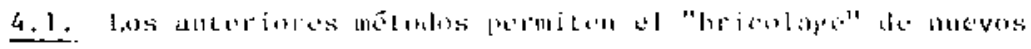

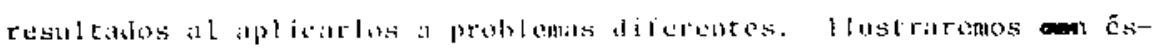

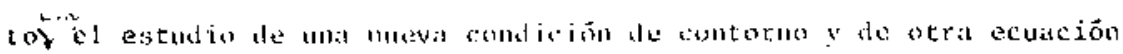
no lineal:

4.1,1. Dado $\gamma$ grafo maximale monótono de $\mathbb{R}^{2}$ con $0 \in \gamma(0), m>0$ y $\Omega$ abierto regular acotado de $\mathbb{R}^{N}$, conside remos el problema

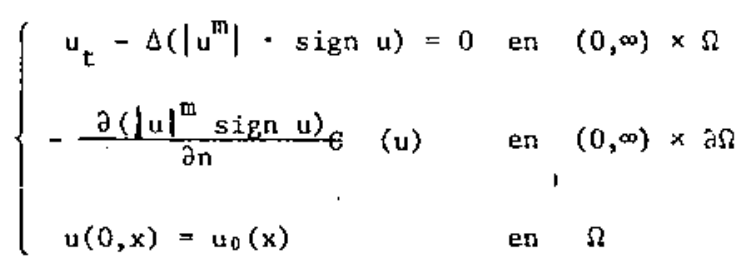

La existencia de suluciones (incegraies) en $\mathrm{L}^{\prime}(\Omega)$ se tiene gracias a un resultado de Benilan [2] y varios criterios de comparación se pueden encontrar en I. Díaz [10].

A fin de estudiar la extinción en t.f. observemos sie si suponemos $u_{0} \geq 0$ y $Y$ univoco (por simplicidad), se tiene "formalmente" que

$$
\frac{d}{d t} \int_{\Omega} u=\int_{\Omega} \frac{d u}{d t}=\int_{\Omega} \Delta u^{m}=\int_{\partial \Omega} \frac{\partial u^{m}}{\partial n}=-\int_{\partial \Omega} Y(u)
$$

por 10 que si $Y^{-1}(0)$ es un cerrado que no se reduce al $\{0\}$, es posible obtener soluciones que conservan su \|\|$_{L^{1}}$ en el tiempo. Más concretamente se tiene que si $K \in \mathbb{R}^{+}$es tal que $Y(K)=0$ entonces la furción $u(t, \cdot) \equiv K$ es la solución de (13) correspondiente a $u_{p}(x) \equiv k$ y está claro que u no se extingue. 
E1 siguiente resultado muestra la sxtinción baju una hi pótesis mâs fuerte que la de compatibilidad $\left(\gamma^{-1}(0)=0\right)$ :

Teorema 5 .

Supuestos $0<$ in $<1 \quad Y$ grafo maximal monótono du $\mathbf{R}^{2}$ con $0 \in \gamma(0)$ y verificanda:

(14) existe $c>0$ tal que $\left|\gamma^{0}(r)\right| \geq c|r|^{m}$ para todo $r \in D(\gamma)$ entonces para todo $u_{0} \in L^{\infty}(\Omega), \underline{1 a}$ soluciốn $\mathrm{u} \in \mathrm{C}\left([0, \infty): \mathrm{I}^{\prime}(\Omega)\right)$ de (13) se extingue en tiempo finito.

\section{Demostración.}

Por los resultados de comparacion, se puede suponer $u_{0} \geq 0$ sin pêrdida de generalidad. Como en 2.2 , bastará construir una supersolución $\hat{u}$ extinguiêndose en t.f. Definamos como antes $\hat{u}(t, x)=f(t):(g(x))^{1 / m}$ donde ahora $g(x)$ se coma de forna que

$$
\begin{array}{cc}
\exists \lambda>0 & \text { tal que } g+\lambda \Delta g=0 \text { en } \Omega \quad y \\
-\frac{\partial g}{\partial n}=G . g & \text { en } \partial \Omega .
\end{array}
$$

Gracias a un teorema debido a Krein-Rutman (véase p.e. Courant-Hilbert [7]), se sabe que $\exists \theta>0$ tal que $0<\theta \leq g(x) \leq 1$ en $\bar{\Omega}$.

$$
\text { Finalmente escojamos } f(t) \text { como en } 2.2 \text {. Las propie- }
$$

dades a), b), c) y d) de allí siguen siendo vâlidas para nuestra super solución $\hat{u}$, pero la condición c) (e.d, la información en $(0, \infty) \times 2 \Omega)$ no hasta para poder aplicar los resulcados de comparación. Una telalaciôn suficiente para ésto (vêase díaz [10])

$$
-\frac{\partial \hat{u}^{m}}{\partial \mathrm{n}}(t, x) \leq \gamma^{0}(\hat{u}) \text { en }(0, \infty) \times \partial \Omega
$$


siendo $Y^{0}(s)$ elemento de norma mínina del- conjụnto $Y(s)$. Ahora bien, es claro que

$$
\begin{aligned}
-\frac{\partial \hat{u}^{\mathrm{m}}}{\partial \mathrm{n}}(t, x) & =-f(t)^{m} \cdot \frac{\partial g}{\partial \mathrm{n}}(\mathrm{x})=\mathrm{f}^{\mathrm{m}}(\mathrm{t}) \cdot \mathrm{C} \cdot \mathrm{g}(\mathrm{x}) \leq \mathrm{c} \cdot(\hat{\mathrm{u}}(\mathrm{t}, \mathrm{x}))^{\mathrm{m}} \leq \\
& \leq \gamma^{0}(\hat{\mathrm{u}}(\mathrm{t}, \mathrm{x})) \text { en }(0, \infty) \times \partial \Omega
\end{aligned}
$$

con lo que concluye la demostración $\left({ }^{4}\right)$

Es fácil ver que la hipótesis (14) no puede ser mejorạ da (sustancialmente) si se utiliza una supersolución de variables separables. Seria interesante entances emplear otros mëtodos o conocer si (14) es también necesaria.

4.1.2. Otro problema motivo de la atención de varios autores es el dajo por

$$
\begin{cases}u_{t}-\Delta_{p}|u|^{m} \cdot \operatorname{sign} u=0 & \text { en }(0, \infty) \times \bar{\Omega}(\bar{\Omega} \text { acota.10) } \\ u=0 & \text { en }(0, \infty) \times \partial \Omega, p>1 \\ u(0, x)=u_{0}(x) & \text { en } \Omega\end{cases}
$$

Diferentes tearemas de existencia han sido dados por Bamberger [1] Kalahsnikov [20\} Benilan [3]... . En concreto se puede asegurar lá existencia de una única solución (integra1) u $6 \mathrm{C}\left([0, \infty): \mathrm{L}^{1}(\Omega)\right.$ ) supuesto uo $\in L^{1}(\Omega)$. Varios criterios de comparación pueden encontrar se en Benilan [3], Herrero $[18], \ldots$ y en lo que sigue, Ios supondremos vâlidos para nuestras condiciones $(m>0$ y $p>1)$.

( $\left.{ }^{4}\right)$ El autor agradece la sugerencia de $\mathrm{J}$. Hernändez sobre la conside ración del problena (13) mediante las técnicas 2.2. 
Una primera observación en el estudio de la extinción Dara (15) es que el Teorema $3, b$ ) puede ser aplicado a $q=p-l$ por 10 que si

$$
m \cdot(p-1)>1
$$

no puede verificarse la propiedad. Por otra parte es fácil buscar con traejemplos para el caso $m \cdot(p-1)=1$ (tómese p.e. $m=1, p=2$ y se obtendrá la ecuación lineal del calor). Veamos a continuación un resultado positivo:

\section{Teorema 6.}

Dado $\Omega$ acotado y $v_{0} \in \mathrm{L}^{\infty}(\Omega), \underline{1 \text { a solución }}$ $u \in C\left([0, \infty): L^{\prime}(\Omega)\right)$ de (15) se extingue en tiempo finito en los siguientes casos
a) $p \geq 2 \underline{y} m \cdot(p-1)<1$
b) $m \cdot(p-1)<1<\left(p^{*-1}\right) m$ (sieudo $p^{*}$ dado por $\frac{1}{p^{*}}=\frac{1}{p}-\frac{1}{N} \quad \underline{s_{i}} \frac{1}{p}-\frac{1}{N}>0 \quad \underline{y} p^{*}=+\infty$ en otro caso).

Demostración.

De nuevo basta supaner $u_{0} \geq 0$ y explicitar la supersolución $\tilde{u}(\mathrm{r}, \mathrm{x})$. Sea $\tilde{\mathbb{m}}=\mathrm{m}(\mathrm{p}-1)$. Para el caso a) se define $\tilde{u}(t, x)=\tilde{f}(t)(\tilde{g}(x))^{1 / m}$, siendo ahora elegida $\tilde{g}$ de forma que

$$
\exists \lambda>0 \text { tal que } \tilde{g}(x)+\lambda \Delta_{p} \vec{g}(x)=0 \text { en } \Omega \text {. }
$$

Gracias a que la inclusión de $w_{0}^{2}+(\Omega)$ en $L^{2}(\Omega)$ es compacta, se puede aplicar un resultado de Bamberger [1] asegurando la existencia de una tal función $\tilde{\mathrm{g}}$, verificando además que

$$
\exists \theta>0 \text { tal que } 0<\theta \leq \widetilde{g}(x) \leq 1 \text { en } \bar{\Omega} \text {. }
$$


La función $\tilde{\mathrm{f}}$ es escogida como solución del publema

$$
\begin{cases}\overline{\mathrm{f}}^{\prime}(\mathrm{r})+\frac{(\tilde{\mathrm{f}}(\mathrm{t}))^{\tilde{m}}}{\lambda}=0 & \\ \tilde{\mathrm{f}}(0)=\mathrm{f}_{0} & \left(\mathrm{f}_{0} \cdot \theta^{1 / \mathrm{m}} \geq\left\|\mathrm{u}_{0}\right\|_{\mathrm{L}} \infty\right)\end{cases}
$$

Es claro entonces que $\tilde{u}$ se extingue en $t, f$. y además verifica que

$$
\begin{aligned}
\tilde{u}_{t}-\Delta_{p} \tilde{w}^{m} & =\tilde{f}^{\prime}(t)(\tilde{g}(s))^{1 / m}-\tilde{E}(t)^{\tilde{m}} \Delta_{p} g(x)- \\
& \geq f^{\prime}(t) g(x)+\frac{f(t)^{\tilde{m}}}{\lambda} g(x)=0
\end{aligned}
$$

El resto de las condiciones son igualmente satisfecha al igual que en 2.2. Para el caso b) se define $\tilde{u}(t, x)=\breve{f}(t) \breve{g}(x)$ donde ahora. $B$ debe satisfacer

$$
\exists \lambda>0 \text { tal que } \tilde{g}(x)+\lambda \Delta_{p} \tilde{g}(x)^{m}=0 \text { en } \Omega
$$

En este caso la hipótes is $1<\left(\mathrm{p}^{\star-1}\right) \mathrm{m}$ asegura la aplicabilidad del resultado de Bamberger $[L]$, siendo el resto de los detalles similares al caso a).

El apartado b) había sido demostrado por Bamberger [1] mediante técnicas similares a 2.1. Estos métodos son tambiên aplicables a $\Omega=\mathbb{R}^{N}$ (vêase Herrero-väzquez [19]).

4.2. Es interesantes e1 estudio de aquellos problemas en los que se dan simultaneamente las propiedades de extinción finita y de propagasión finita de las señales.

En el caso de modelos de difusión ( $\sin$ términos de absorción) no son conocidos resultados sobre esta coincidencia, y sin embargo existen resultados concretos que aseguran la imposibilidad de 
que se realicen a ja vez. (Véase, por ejemplo el Teorema 2 junto con el Teorema l de T. Míaz [lo]). Fistó laz sido el punto de partida de Ja clasificas ión de los modelos de difusión del calor (en Jcontos. râa-

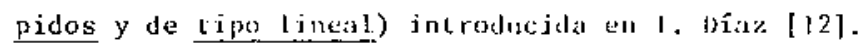

Cuando el modelo incluye términos de absioción âdecuar. dos la situación es enteranenc diferente produciéndose la simultaneidad de diclus fenổmenos e incluso otra propiedad aún más fuerte que es conocida como propiedad de contracción instantanea del soporte. (Pó ra mäs referencias vĕase p.e. Herrero [17] y la comunicación de este autor en el presente Congreso). 


\section{Referencias.}

[1] A. Bamberger, "Etude d'une equation doublement non linea1re", J. Functional Analysis 24, 148-155. 1977

[2] Ph. Benflan, "Equations d'evolution dans un espace de Banach queliconque et applications". Tesis, Universidad de orsay, 1972 .

[3] Ph. Benilan, "Operateurs accretifs et semf-groups dans les espaces $L^{p}, 1 \leq p \leq+\infty "$. Publications de $L^{\prime}$ Univ, de Besancon. 1977 .

[4] Ph. Benilan y M.G. Crandal1, "The cont1nuous dependence on $\phi$ of solution of ${ }^{u}-\Delta \phi(u)=0 "$. T.S.R. Mathematics Research Center. Univ. of Wisconsin-Madison (Aparecera)

[5] J.C. Berryman y C.J. Holland, "Stability of the separable solution for fast diffuslon". Aparecerä en Arch. Rational Mech. Anal.

[6] H. Brezis, "Monotone operators, non linear semigroups and applications", Proc. Int. Congress Mth. Vancouver. 1974.

[7] R. Courant y D. Hilbert, "Methods of Mathematcal Physics", New York. Interscience 1953.

[8] G. Dỉaz Dlaz, "Extinción fintta para ecuactones parabólicas con no linealidades sobre operadores ellpticos", (Aparecerá en las actas de las vi a Jornadas Matematicas Hispano-Lusas. Santander 1979.

[9] G. Diaz DIaz y J.I. DLaz DIaz, "Finite extinction time for a class of nonlinear paraboltc equations", Aparecerá en Contr. in Part. Diff. Equations.

[10] J.I. DIaz Blaz, "Solutions with compact' support for some dege nerate parabolic problems", Aparecerá en J. of Non. Anal. 
[11] J.I. Díaz Diaz, "Anulación de soluciones para ciertos problemas parabólicos no lineales", Rev. Real Acad. Ci. Exactas, Fis, y Nat. Madrid LXXII, 613-619, 1979 (y artículo detallado a aparecer en la misma revi:., s).

[12] J.I. Díaz Díaz, "Propiedades cualitativas de ciertos problemas parabólicos no 1 ineales: Una clasificación para los mo delos de difusión del calor", Aparecerá en Rev. Real Acad. Ci. Exactas, Fislicis y Nar. Madrtd.

[13] J.I. Dỉaz Díaz, Trabajo en realización.

[14] J.I. DIaz Díaz y M.A. Herrero Garcia, "Compact support properties for somte elliptic and parabolic equations artsing In the theory of Non Newtonian fluids", Aparecera.

[15] G. Duvaut y J.L. Lions, "Les inequations en Mecanique et en Physique", Dunod. Paris 1972.

[16] L.C. Evans, Resultado no publicado.

[17] M.A. Herrero García, "Sobre el comportamiento de las soluciones de algunos problemas parabólicos no lineales", Aparecerá en Rev. Real Acad. Ci. Exactas, Fis. y Nat. Madrid.

[18] M.A. Herrero Garcia, "Comportamiento de las soluciones de ciex cos problemas no lineales sobre dominios no acotados", Tesis, Univ. Complutense de Madrid, 1979.

[19] M.A. Herrero García y J.L. Vázquez Suärez, Trabajo en redacciín

[20] A.S. Kalashnikov, "On a non-linear equation arising $f$ th the theory of non-stationary filtration", Actas deI Semtnar10 Y. G. Petrowski. Vo1. 4, 137-146. Izd, Moscovsk. UnIv. 1978 .

[21] A. Majda, "Disappearing solutions for the dissipative wave eqs.". Indiana Univ. Math. Jur. 24, 1119-1133, 1975.

[22] L. Veron, Coerctuite et proprietes regularisantes des setti-grou pes dans les espaces de Banach", Pub1. Math. Fac. Sc1. Besancon, n우 3. 1977. 